\title{
Insomnia theory and assessment
}

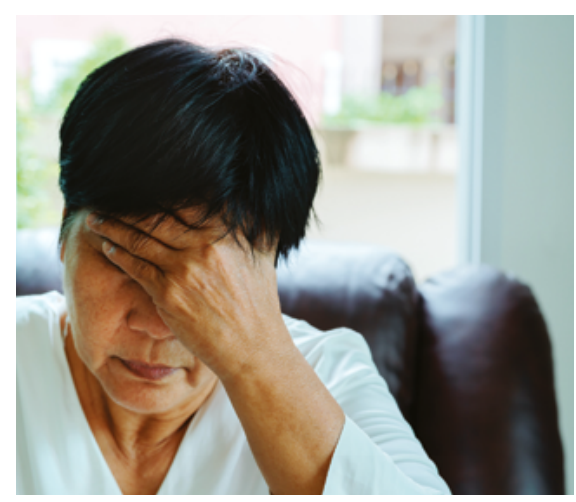

CPD

Natalie A Grima, Bei Bei,

Darren Mansfield

\section{Background}

Insomnia is a common condition affecting individuals of various ages. It is diagnosed on the basis of a selfreported complaint of poor sleep quality concomitant with daytime disturbances. If left untreated, insomnia is associated with a number of adverse health outcomes.

\section{Objectives}

The aim of this article is to review key diagnostic criteria, theories and assessment of insomnia.

\section{Discussion}

Insomnia may be precipitated by stressful events. Unhelpful strategies employed by the individual to remedy sleep can perpetuate insomnia symptoms even after the stressful event subsides. Insomnia is often undiagnosed and undertreated, which is concerning given that untreated insomnia associated with a number of negative health outcomes. Diagnosis and treatment planning can be facilitated using self-report measures.

\section{Definition, diagnosis and clinical features}

Insomnia is characterised by self-reported poor sleep quality or inadequate sleep despite adequate sleep opportunity and sleep-conducive environment, and it is accompanied by impairment to daytime function. There are two separate classification systems for sleep disorders, the International classification of sleep disorders, 3rd edition (ICSD-3) ${ }^{1}$ and the Diagnostic and statistical manual for mental disorders, 5th edition (DSM-5). ${ }^{2}$ Table 1 outlines the similarities and differences between the two classification systems. The diagnosis of insomnia disorder is comparable across the two classification systems, but for patients who do not meet the full criteria, the two systems have different labels.

According to ICSD-3, a diagnosis of insomnia is present when a patient presents with three criteria, with the chronicity of symptoms used to specify the diagnosis (Table 1). The criteria include:

- a subjective complaint of poor sleep quality, which may include difficulties initiating sleep, fragmented sleep characterised by recurrent nocturnal arousals manifesting in difficulties with maintaining sleep throughout the night, or waking up earlier than desired

- sleeping difficulties despite adequate opportunity for sleep
- distress or some impairment in daytime function occurring as a result of sleeping difficulties.

\section{Assessment}

A diagnosis of insomnia is based on the patient's self-report. Objective sleep assessment is not necessary for diagnosis, although polysomnography is diagnostically useful in patients with suspected cormorbid sleep disorder. It is important to note that both the ICSD-3 and DSM-5 have steered away from the classification of primary and secondary insomnia (ie insomnia comorbid with another disorder). This change reflects a growing awareness that when insomnia symptomatology arises in the context of another condition, it may evolve into a separate disorder that may not necessarily resolve after successful treatment or resolution of the first condition. This is pertinent in patients with a psychiatric history, given the frequency with which insomnia and psychiatric disturbances co-occur. ${ }^{3}$

Diagnosis of insomnia is facilitated by the use of self-report measures to characterise the sleep disorder and elucidate comorbidities (eg mood, circadian rhythm abnormality), which can assist with selecting 
the right treatment (Box 1). ${ }^{4}$ In patients who are not responding to pharmacological intervention or patients in whom pharmacologic intervention is contraindicated (eg substance use history), specialist treatment is best undertaken by a multidisciplinary team that includes collaboration between a sleep physician, psychologist and general practitioner in concert with the patient.

\section{Prevalence}

Taking into account recent diagnostic changes, the prevalence of insomnia disorder is estimated to be at $11.3 \%$. In addition, about one-third of adults (30-36\%) may have some insomnia symptoms (eg poor sleep quality and non-restorative sleep) without meeting full diagnostic criteria. ${ }^{5,6}$ Women tend to have insomnia more commonly than men, with insomnia more commonly reported among young and middle-aged adults than the elderly.

\section{Clinical features and pathogenesis}

Patients with insomnia usually describe sleep as 'non-refreshing' or 'non-restorative', attributing daytime impairments such as fatigue and irritability to poor sleep. ${ }^{7}$

They also tend to underestimate their actual time asleep and overestimate the wakefulness and time to fall asleep, ${ }^{8}$ and are more likely to identify themselves as being awake even though polysomnography indicate they are asleep; this phenomenon is referred to as sleep state misperception. ${ }^{9}$

Insomnia is often accompanied by hyperarousal, which is underpinned by physiologic, psychological, behavioural and cognitive factors. ${ }^{10}$ Psychological and behavioural hyperarousal may be caused by effort to try to sleep (ie sleep effort). Patients may 'try hard to sleep', adopt rigid nightly routines, and engage in avoidance and safety behaviours (eg cancelling a work meeting because of poor sleep; increasing time in bed to make up for lack of sleep) ${ }^{10}$ Individuals start planning their lives around sleep and ruminating about upcoming events that require a deviation from their usual routine in fear of a poor night sleep. ${ }^{7}$ Not unexpectedly, sleep effort usually results in increased emotional and cognitive arousal, which is not conducive to sleep. Patients may also develop unhelpful thoughts and beliefs (eg 'If I can't sleep within the next 15 minutes, I will not sleep'), and catastrophise about the consequence of poor sleep (eg 'If I don't sleep, my day is ruined'). Patients' cognitions and beliefs regarding sleep often present themselves in the language and emotions used, which can assist with the formulation of therapeutic intervention to address sleep. ${ }^{11}$

Various insomnia models exist, ${ }^{12}$ though the original theoretical model referred to as the Spielman Model ${ }^{13}$ has been expanded to include four factors $^{8}$ (referred to as the 4P model) that are thought to underpin insomnia: predisposing factors (eg characterological features such as an anxious temperament, and perfectionist qualities), ${ }^{14}$ precipitating factors (eg stressful events), perpetuating factors (eg behaviours employed by the individual to compensate for sleeplessness) and Pavlovian conditioning. Perpetuating factors include staying in bed while awake, daytime napping, spending excessive time in bed, and dependence on alcohol and other sedatives to assist with sleep initiation. ${ }^{14}$ In the context of insomnia, Pavlovian conditioning refers to the elicitation of arousal in response to the bedroom environment (eg patients may report, 'I'm sleepy but as soon as I hit the pillow I'm awake').

\section{Table 1. Comparison of the ICSD-3 and DSM-5 classifications of insomnia}

\begin{tabular}{ll}
\hline ICSD-3 insomnia classifications & DSM-5 insomnia classifications \\
Chronic insomnia & Insomnia disorder - similar to ICSD-3 \\
Short-term insomnia - No DSM-5 & diagnosis of chronic insomnia \\
equivalence & Other specified insomnia disorder
\end{tabular}

\section{Other insomnia disorder}

\section{Diagnostic similarities for ICSD-3 chronic insomnia and DSM-5 insomnia disorder}

1. A self-reported complaint of poor sleep quality including one of the following:

- difficulties initiating sleep

- difficulties maintaining sleep

- waking up earlier than desired.

2. Sleep difficulties occur despite adequate sleep opportunity.

3. Impaired sleep produces deficits in daytime function.

Note: The DSM-5 emphasises sleep disturbance must cause clinically significant distress or impairment across personal, vocational, behavioural, social, educational or other areas of functioning.

4. Sleep difficulty occurs three nights per week and is present for three months.

Conditions:

- ICSD-3 - Symptoms lasting <3 months can be coded 'Short-term insomnia'.

- DSM-5 - Symptoms lasting <3 months, but meeting all other criteria, can be coded 'Other specified insomnia disorder'.

\section{Outdated insomnia diagnosis and terminology}

- Acute insomnia

- Stress-related insomnia

- Transient insomnia

- Primary insomnia

- Secondary insomnia

- Comorbid insomnia

DSM-5, Diagnostic and statistical manual for mental disorders, 5th edition; ICSD-3, International classification of sleep disorders, 3rd edition 


\section{Box 1. Questions and sleep measures used to establish a diagnosis}

\section{Sleep schedule}

- The Consensus Sleep Diary ${ }^{27}$ (available freely online) is the most reliable way to determine sleep schedule and sleep variability. Particulars include bedtime, rise time, time spent sleeping during the night, nocturnal awakenings, time taken to get to sleep, napping (frequency and duration).

- The patient's daily preferences for rest and activity can be used to establish morningness/ eveningness preferences (ie 'early bird/night owl').

\section{Comorbidities}

- It is important to determine behaviours during sleep, such as snoring, witnessed apnoeas, restless legs, sleep walking, nightmares. Consider medical, psychiatric (use the K10) and lifestyle comorbidities (eg long work days, nightshift).

\section{Patient's perception}

- How does the patient view their sleep problem? Do they attribute daytime functioning impairment to poor sleep, or are there other factors?

- Measures such as the Insomnia Severity Index and Epworth Sleepiness Scale (available freely online) can be used to rate insomnia severity and daytime somnolence.

\section{Duration and frequency}

- Establish chronicity of condition and previous episodes of poor sleep and past treatment.

\section{Sleep specialist services in Australia}

- In Australia, sleep services can be found by visiting the Australasian Sleep Association service directory (www.sleep.org.au).

In the event of a precipitating event, predisposed individuals are at risk of poor sleep. ${ }^{10}$ Pavlovian conditioning (ie classical conditioning) is thought to have a critical role in perpetuating insomnia. Attempts to sleep despite being awake result in the bedroom environment becoming paired with an awake state. Over time, the bed is no longer associated with sleep; rather, the bed environment triggers an 'awake' state. These perpetuating factors are often described as 'maladaptive coping strategies' because attempts to increase sleep opportunity will inevitably result in a mismatch between sleep opportunity and ability, which will increase the likelihood that the individual will be awake during the sleep period. ${ }^{12}$

More recently, the Spielman model has been expanded by the neurocognitive model of insomnia (Figure 1) proposed by Perlis et $\mathrm{al}^{8}$ to include the insomnia concept of hyperarousal. ${ }^{12,14}$ Arousal can be experienced somatically (eg 'feeling wired') or cognitively (eg a racing mind), and increased cortical activation can be measured electroencephalographically. ${ }^{12}$ The arousal is thought to promote heightened sensory and informationprocessing capacity and memory formation. Heightened information processing during sleep may blur the distinction between sleep and wakefulness, leading to the phenomena of sleep state misperception. ${ }^{15}$ Similarly, heightened memory formation during sleep onset and nocturnal arousals are thought to interfere with the subjective experience of uninterrupted sleep. ${ }^{15}$

\section{Societal and personal consequences of insomnia}

In Australia, it was estimated that during the 2016-17 financial year that insufficient sleep due to sleep disorders such as insomnia contributed to other conditions - such as heart disease, stroke, diabetes and depression - costing the Australian health system $\$ 1.24$ billion. ${ }^{16}$ The direct healthcare costs associated with the treatment of insomnia were estimated to be $\$ 118.7$ million in $2010 .^{17}$ The indirect costs of insomnia manifesting in reduced employee productivity, higher absenteeism and workplace accidents were estimated to be $\$ 1.5$ billion in 2010. ${ }^{17}$

At an individual level, insomnia is associated with a range of physiological and psychological adverse outcomes. At a physiological level, insomnia is associated with measurable alterations in cortisol level, increased metabolic activity and neuro-immunological functioning, as well as increased blood pressure and high-frequency electroencephalography activity. ${ }^{15,18}$ A comprehensive review of the current literature now indicates that insomnia, when accompanied by short sleep duration, is associated with an increased risk for hypertension, coronary heart disease, heart failure and recurrent acute coronary syndrome, ${ }^{19}$ a view shared by others. ${ }^{20}$ The mechanisms are thought to be multifactorial and mediated by the dysregulation of the hypothalamicpituitary axis, increased sympathetic tone and inflammation. ${ }^{19}$ Cognitively, patients with insomnia display performance deficits in some facets of executive functioning, including multitasking and working memory. ${ }^{21,22}$ In patients with insomnia, poorer sustained attention is associated with reduced quality of life, ${ }^{22}$ and patients with insomnia generally report reduced quality of life irrespective of cognitive functioning. ${ }^{23}$

Untreated insomnia results in a greater risk of psychiatric disorders. ${ }^{24}$ Insomnia may precede a psychiatric condition, or it may emerge at the same time. ${ }^{10,24}$ Insomnia disorder and symptoms may occur across a number of psychiatric conditions including, but not limited to, major depressive and bipolar disorder, anxiety, post-traumatic stress disorder, obsessive-compulsive disorder, substance use disorder and psychosis. ${ }^{25,26}$

\section{Conclusion}

Insomnia is a multifaceted sleep condition that affects a large proportion of individuals and results in self-perceived dissatisfaction with sleep with concomitant effects on daytime functioning. Early identification of the contributory factors underpinning insomnia may shed light on precipitating factors that, if addressed, may result in improvement in sleep. In some individuals, insomnia symptoms may persist after the precipitating event and comorbid conditions have resolved, 
resulting in insomnia disorder. Untreated insomnia is associated with a plethora of adverse health and psychological outcomes, with sleep problems unrecognised. Assessment of insomnia can be achieved by self-report measures that can assist with diagnosis and guide treatment planning.

\section{Key points}

- Insomnia is a common sleep condition that can persist for decades if left untreated.

- Insomnia disorder is associated with adverse medical and psychiatric risk factors, which can exacerbate other conditions.

- Insomnia may emerge in the context of a psychiatric condition; in this case, treatment of insomnia in addition to treatment of the psychiatric condition is necessary.

\section{Authors}

Natalie A Grima BSc, GradDipPsych, DPsych(ClinNeuro), clinical neuropsychologist, Monash Institute of Cognitive and Clinical Neurosciences, Monash School of Psychological Sciences, Faculty of Biomedical and Psychological Sciences, Monash University, Vic

Bei Bei DPsych(Clinical), PhD, NHMRC Health Professional Research Fellow, Monash Institute of Cognitive and Clinical Neurosciences, Monash School of Psychological Sciences, Faculty of Biomedical and Psychological Sciences, Monash University, Vic

Darren Mansfield MBBS, PhD, Associate Professor, Adjunct School of Psychological Sciences, Monash University, Vic; Deputy Director, Monash Lung and Sleep, Monash Health, Vic. darren.mansfield@ monashhealth.org

Competing interests: NAG is a clinician in a multidisciplinary team for the treatment of insomnia and circadian rhythm sleep disturbances.

Funding: None.

Provenance and peer review: Commissioned, externally peer reviewed.

\section{References}

1. American Academy of Sleep Medicine. International classification of sleep disorders 3rd edn. Darien, IL: American Academy of Sleep Medicine, 2014.

2. American Psychiatric Association. Diagnostic and statistical manual of mental disorders, 5th edn. Arlington, VA: American Psychiatric Association, 2013.

3. Riemann D. Insomnia and comorbid psychiatric disorders. Sleep Med 2007;8 Suppl 4:S15-20. doi: 10.1016/S1389-9457(08)70004-2.

4. Cunnington $D$, Junge M. Chronic insomnia: Diagnosis and non-pharmacological management. BMJ 2016;355:i5819. doi: 10.1136/bmj.i5819.

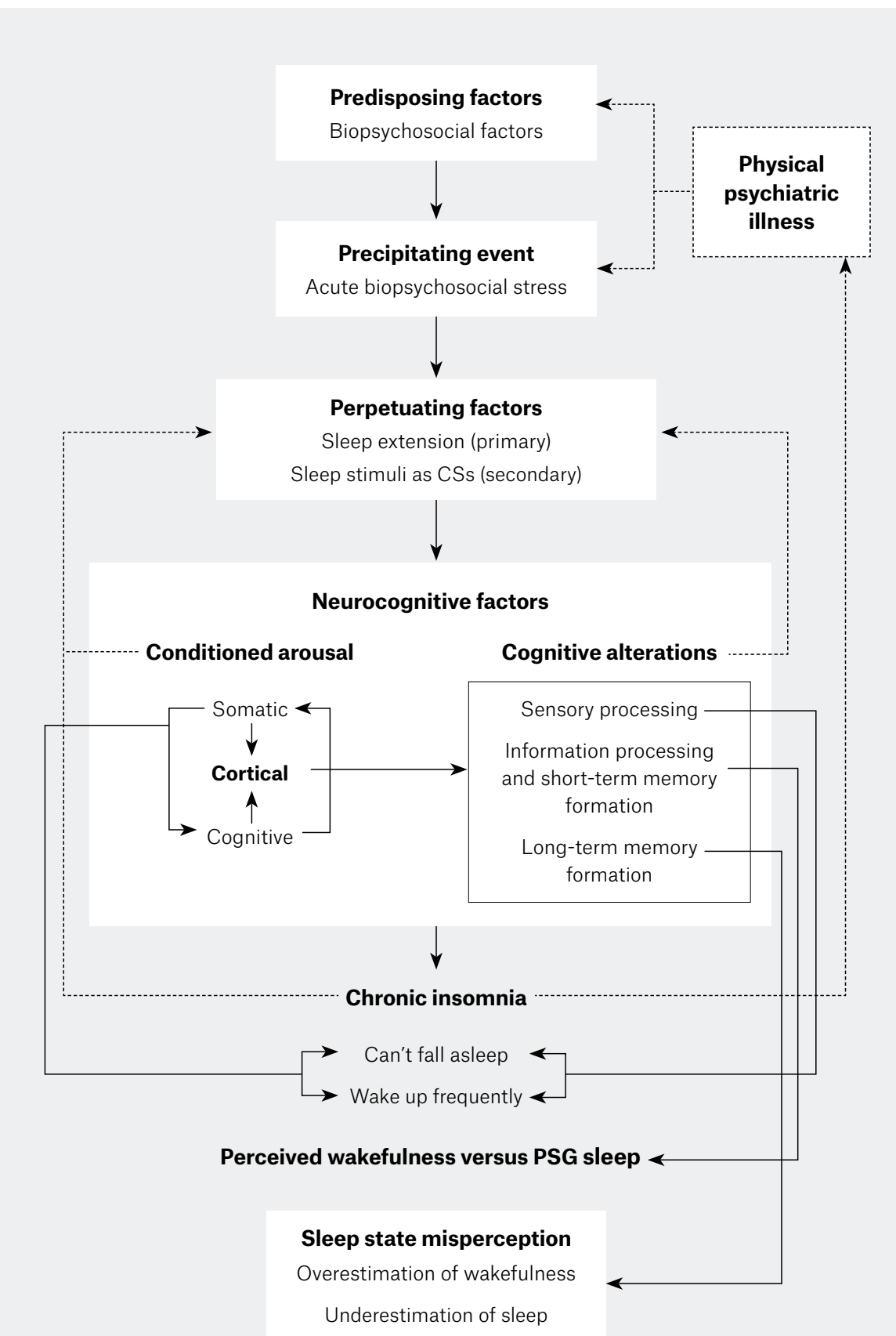

Figure 1. The neurocognitive model of insomnia. Increased time in bed, episodes of wakefulness and distress in bed triggered by the precipitating event become associated with the bedroom environment. Over time, these contextual and temporal stimuli result in the sleep environment being conditioned with wakefulness resulting in heightened arousal and altered cognitive alterations, referred to as hyperarousal, which inhibits sleep. Hyperarousal results in the overestimation of wakefulness and sleep/wake misperception.

CSs, conditioned stimuli; PSG, polysomonography

Reprinted from Perlis M, Ellis JG, Kloss JD, Riemann DW. Etiology and pathophysiology of insomnia. In: Kryger MH, Roth T, Dement WC, editors. Principles and practice of sleep medicine. 6th edn. Philadelphia, PA: Elsevier, 2017; p. 774, with permission from Elsevier. 
5. Sleep Health Foundation. Asleep on the job: Costs of inadequate sleep in Australia. Blacktown, NSW: Sleep Health Foundation, 2017. Available at www. sleephealthfoundation.org.au/files/Asleep_on_the job/Asleep_on the_Job_SHF_report-WEB_small.pdf [Accessed 1 March 2019].

6. Adams RJ, Appleton SL, Taylor AW, et al. Sleep health of Australian adults in 2016: Results of the 2016 Sleep Health Foundation national survey. Sleep Health 2017;3(1):35-42. doi: 10.1016/j. sleh.2016.11.005.

7. Samaranayake CB, Fernado AT. Insomnia classification, features, diagnosis, and evaluation. In: Mansfield D, Antic NA, Rajaratnam SMR, Naughton M, editors. Sleep medicine. Melbourne: IP Communications, 2017; p. 297-300.

8. Perlis ML, Giles DE, Mendelson WB, Bootzin RR, Wyatt JK. Psychophysiological insomnia: The behavioural model and a neurocognitive perspective. J Sleep Res 1997 Sep;6(3):179-88.

9. Harvey AG, Tang NKY. (Mis)perception of sleep in insomnia: A puzzle and a resolution. Psychol Bull 2012;138(1):77-101. doi: 10.1037/a0025730.

10. Manber R, Carney C. Treatment plans and interventions for insomnia: A case formulation approach. New York: The Guilford Press, 2015.

11. Cunnington D, Junge MF, Fernando AT. Insomnia: Prevalence, consequences and effective treatment. Med J Aust 2013;199(8):36-40. doi: 10.5694/ mja13.10718.

12. Perlis M, Ellis JG, Kloss JD, Riemann DW. Etiology and pathophysiology of insomnia. In: Kryger $\mathrm{MH}$, Roth T, Dement WC, editors. Principles and practice of sleep medicine. 6th edn. Philadelphia, PA: Elsevier, 2017; p. 769-84.

13. Spielman AJ, Caruso LS, Glovinsky PB. A behavioral perspective on insomnia treatment. Psychiatr Clin North Am 1987;10(4):541-53.

14. Yang CM, Spielman AJ, Glovinsky P. Nonpharmacologic strategies in the management of insomnia. Psychiatr Clin North Am 2006:29(4):895-919.

15. Riemann D, Spiegelhalder K, Feige B, et al. The hyperarousal model of insomnia: $A$ review of the concept and its evidence. Sleep Med Rev 2010;14(1):19-31. doi: 10.1016/j. smrv.2009.04.002.

16. Hillman D, Mitchell S, Streatfeild J, Burns C, Bruck D, Pezzullo L. The economic cost of inadequate sleep. Sleep 2018;41(8). doi: 10.1093/ sleep/zsy083.

17. Sleep Health Foundation. Re-awakening Australia: The economic cost of sleep disorders in Australia. Blacktown, NSW: Sleep Health Foundation, 2011. Available at www.sleephealthfoundation.org.au/ public-information/special-reports/reawakeningthe-nation.html [Accessed 1 March 2019].

18. Bonnet MH, Arand DL. Hyperarousal and insomnia: State of the science. Sleep Med Rev 2010;14(1):9-15. doi: 10.1016/j.smrv.2009.05.002.

19. Javaheri S, Redline S. Insomnia and risk of cardiovascular disease. Chest 2017;152(2):435-44 doi: 10.1016/j.chest.2017.01.026.

20. Spiegelhalder K, Scholtes C, Riemann D. The association between insomnia and cardiovascular diseases. Nat Sci Sleep 2010;2:71-80.

21. Shekleton JA, Rogers NL, Rajaratnam SMW. Searching for the daytime impairments of primary insomnia. Sleep Med Rev 2010;14(1):47-60. doi: 10.1016/j.smrv.2009.06.001.

22. Shekleton JA, Flynn-Evans EE, Miller B, et al. Neurobehavioral performance impairment in insomnia: Relationships with self-reported sleep and daytime functioning. Sleep 2014;37(1):107-16. doi: $10.5665 /$ sleep.3318.
23. Léger D, Guilleminault C, Bader G, Lévy E, Paillard M. Medical and socio-professional impact of insomnia. Sleep 2002;25(6):625-29.

24. Ohayon MM, Roth T. Place of chronic insomnia in the course of depressive and anxiety disorders. J Psychiatr Res 2003;37(1):9-15.

25. Khurshid AK. Comorbid insomnia and psychiatric disorders: An update. Innov Clin Neurosci 2018;15(3):28-32.

26. Cohen ZL, Sharkley KM. Insomnia in psychiatric disorders. In: Attarian HP, editor. Clinical handbook of insomnia. Totowa, NJ: Humana Press, 2017; p. 267-81.

27. Carney CE, Buysse DJ, Ancoli-Israel S, et al. The Consensus Sleep Diary: Standardizing prospective sleep self-monitoring. Sleep 2012;35(2):287-302. doi: 10.5665/sleep.1642. 